\title{
Early findings in a pilot study of anterior cervical fusion in which bioabsorbable interbody spacers were used
}

\author{
Alexander R. Vaccaro, M.D., Matthew M. Robbins, B.A., Luke Madigan, M.D., \\ Todd J. Albert, M.D., William Smith, M.D., and Alan S. Hilibrand, M.D. \\ Department of Orthopaedic Surgery, Thomas Jefferson University Hospital, Rothman Institute; \\ Jefferson Medical College, Philadelphia, Pennsylvania; and Western Regional Center for Brain \\ and Spine Surgery, Las Vegas, Nevada
}

\begin{abstract}
Object. In this pilot study the authors assessed the efficacy of bioabsorbable interbody spacers in the treatment of cervical degenerative disease. Metallic cages or interbody spacers have been widely used in the treatment of degenerative and traumatic cervical disease. Bioabsorbable technology has been used to develop a resorbable cage that can eliminate the complications and drawbacks seen with the use of traditional metallic implants. In general clinical practice bioabsorbable implants have shown the ability to degrade safely while demonstrating optimal imaging characteristics as a result of their radiolucency, and these devices eliminate stress shielding by their gradual dissolution.

Methods. This study is a retrospective evaluation of charts and x-ray films obtained in the first eight patients who underwent an anterior cervical decompression and fusion procedure with placement of a bioabsorbable interbody spacer and anterior cervical plate. All patients were treated in one surgeon's practice and had a minimum follow-up period of at least 6 months. At a follow-up interval of approximately 7 months, five patients exhibited an excellent result and three had a good result; no patient was noted to have a satisfactory or poor outcome according to the Odom criteria at their most recent follow-up visit. Seventeen (94\%) of 18 grafted levels appeared to be solidly fused. One patient experienced a perisurgical complication consisting of a symptomatic hematoma, which was successfully drained.

Conclusions. Bioabsorbable interbody spacers appear to be a safe and effective interbody implant in terms of clinical outcome and radiographically confirmed healing.
\end{abstract}

\section{KEY WORDS • cervical fusion • spinal surgery • bioabsorbable implant • interbody spacer}

The use of interbody spacers, cages, or "baskets" for the treatment of cervical degenerative disease originated with the work of Bagby ${ }^{1}$ in the treatment of equine wobblers disease. Recently, circular and trapezoidal interbody spacers made from a myriad of materials have become popular in the management of symptomatic degenerative cervical conditions. Interbody spacers have been shown clinically to stabilize cervical motion segments adequately and to facilitate fusion in this spinal disorder. One of the first metallic circular or side-barrel cage designs was the BAK cervical device. This cage demonstrated a fusion rate of greater than $90 \%$ at a 3-year follow up. ${ }^{9}$ Reports of carbon fiber cages designed in a trapezoidal shape have also noted high fusion rates, with the added benefit of the cages' radiolucence. ${ }^{7}$

Eck, et al., have discussed the advantage of synthetic cervical cage technology in correcting sagittal spinal alignment through the varied selection of cage heights and diameters. The major drawback of contemporary metallic cages, be it cylindrical side-barrel placement or vertical mesh (open to each endplate) designs, is the inability to

Abbreviation used in this paper: rhBMP-2 = recombinant human bone morphogenetic protein-2. assess bone graft incorporation accurately due to the devices' metallic composition. Eck and colleagues ${ }^{6}$ reported on the use of titanium cervical cages in the treatment of sagittal plane deformities. In their study of 66 patients whose mean follow-up period was 33 months, a fusion rate of $78 \%$ was observed, although the authors pointed out the difficulty of assessing fusion radiographically in the setting of a metal implant.

The history of bioabsorbable implants for human application began with absorbable sutures. Today these materials are commonly used for a variety of reconstructive purposes. Orthopedic surgeons routinely use bioabsorbable interference screws in anterior cruciate ligament and arthroscopic shoulder repairs. Resorbable products are also enjoying popularity in fracture fixation. ${ }^{3-5,11}$

A bioabsorbable interbody spacer has many potential advantages compared with contemporary metallic implants. First, bioabsorbable devices allow for a much improved radiographic assessment due to their radiolucency. Another drawback of rigid metallic devices is the mismatch in their modulus of elasticity compared with vertebral bone, which yields the potential for stress shielding and subsidence. By comparison, bioabsorbable implants degrade over time, resulting in gradual dynamization 
across the implant, graft, and vertebral bone, possibly improving the likelihood of successful fusion. In addition, bioabsorbable implants may be capable of serving as carriers for growth factors such as bone morphogenetic proteins without the need for adjunctive internal fixation. ${ }^{8}$

This study is an early retrospective evaluation of an anterior cervical interbody spacer used in the treatment of symptomatic cervical degenerative disease.

\section{CLINICAL MATERIAL AND METHODS}

\section{Patient Population}

A retrospective chart and radiographic review was conducted in the first eight consecutive patients who underwent an anterior cervical decompression and fusion in which a bioabsorbable interbody spacer and anterior cervical metallic plate were used. These patients were treated for symptomatic cervical disc degeneration by a single surgeon (W.S.) between February and March 2003. There were five women and three men with a mean age of 52 and an age range of 46 to 64 years. The diagnoses were radiculopathy in one patient, myelopathy in three, and a combination of radiculopathy and myelopathy in the other four. Patients were given the option of surgical intervention if they failed to improve after conservative treatment consisting of initial rest and immobilization, nonsteroidal antiinflammatory medications, and physical therapy in the setting of objective anterior thecal sac or nerve root compression on magnetic resonance imaging.

\section{Surgical Procedure}

Two patients underwent a single-level, three a twolevel, two a three-level, and one a four-level anterior cervical discectomy and fusion with a Cornerstone HSR (Medtronic Sofamor Danek, Memphis, TN) bioabsorbable spacer (Fig. 1) via a standard left-sided anterior Smith-Robinson approach, followed by anterior metallic plate application. One patient (Case 4) also underwent an adjunctive posterior cervical fusion with lateral mass instrumentation for multilevel cervical stenosis in the setting of symptomatic cervical myelopathy. All patients were treated with adjunctive anterior cervical titanium plate fixation. The interbody spacers or cages were made of a resorbable poly-lactide, that is, 70:30 poly(L-lactide-co-

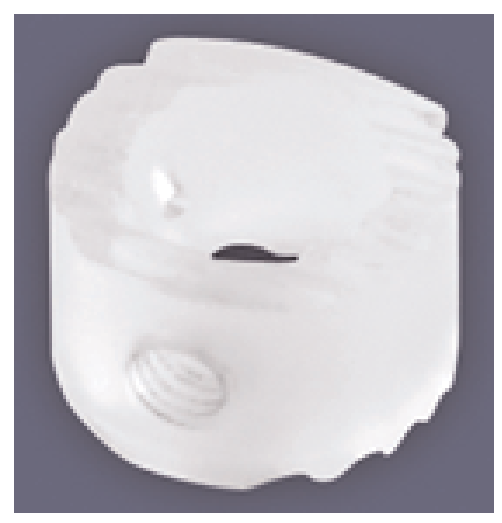

Fig. 1. Photograph showing a bioabsorbable Cornerstone anterior cervical spacer graft.
D,L-lactide), which naturally degrades to carbon dioxide and water. The implants are available in two primary configurations $(14-\mathrm{mm}$ width $\times 11-\mathrm{mm}$ depth and $11-\mathrm{mm}$ width $\times 11-\mathrm{mm}$ depth), and in heights ranging from 5 through $10 \mathrm{~mm}$ in $1-\mathrm{mm}$ increments.

An independent retrospective chart review was performed to gather pertinent patient data including age, sex, duration of symptoms, physical examination findings, types of conservative treatments, surgical approach, level of fusion, use of allograft or autograft bone, use of bone graft extenders or replacements, and use of adjunctive internal fixation as well as length of follow up, smoking history, and patient involvement in either litigation or Worker's compensation claims. Additionally, information was gathered on any postoperative complications, including difficulty swallowing, hoarseness, neck pain, bone graft site discomfort, infection, or hematoma. Imaging studies, including plain x-ray films and computerized tomography scans when available, were reviewed to assess fusion healing or abnormal soft tissue reactions.

Fusion was characterized by a lack of motion between spinous processes on flexion/extension radiographs; absence of a radiolucent space between the graft, interbody spacers (cages), and vertebral endplates; and the presence of bridging by bone trabeculae at the graft/host vertebral endplate interface. Pseudarthrosis was defined as obvious motion between the spinous processes as seen on flexion/extension radiographs, a lucent line at the graft/vertebral endplate boundary, and a lack of osseous trabecular bridging between the graft and host vertebral endplates. Soft tissue changes in the prevertebral space were measured as demonstrated in an illustrative case by using a horizontal line on a lateral plain $\mathrm{x}$-ray film drawn from the anterior margin of the midintervertebral disc level to the posterior esophageal shadow prior to fusion and after cornerstone graft and anterior cervical plate placement (Fig. 2).

To assess the clinical outcome of patients postoperatively, the criteria of Odom, et al. ${ }^{10}$ were used. This scale involves dividing outcomes into four groups, as follows. 1) A poor outcome was defined as the presence of symptoms that were worse or with no improvement after surgical treatment. 2) A patient with symptomatic improvement but persistent physical limitations was designated as having a fair outcome. 3) A good outcome was characterized as the presence of sporadic symptoms that did not limit daily activities. 4) A patient with no symptoms and no limitations in activity was considered to have attained an excellent outcome.

\section{RESULTS}

As shown in Table 1, eight patients underwent an anterior cervical decompression and fusion in which a bioabsorbable anterior cervical interbody spacer and an anterior metallic cervical plate were used. The levels of fusion involved the C2-5 interspaces, the C3-4 interspace, the C3-6 interspaces, the C3-7 interspaces, and the C6-7 interspace in one patient each, and the C5-7 interspaces in three patients. The patient in Case 5 also underwent a posterior C3-7 laminectomy and fusion with lateral mass segmental fixation. The interbody spacer was filled with Grafton demineralized bone matrix putty (Osteotech, Inc., 


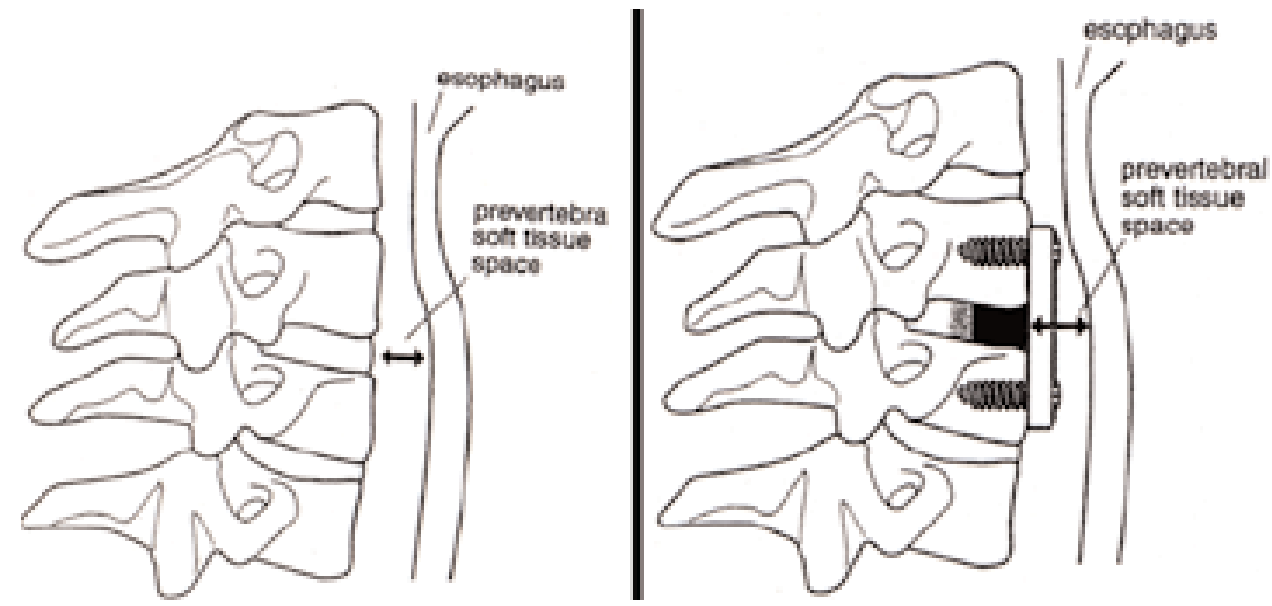

Fig. 2. Left: Drawing showing soft tissue changes in the prevertebral space were measured using a horizontal line drawn from the midintervertebral disk level on a lateral plain x-ray film to the border of the esophageal shadow prior to fusion and placement of Cornerstone graft. Right: Drawing showing how soft tissue changes in the prevertebral space were measured using a horizontal line drawn from the midintervertebral disc level on a lateral plain x-ray film to the border of the esophageal shadow after placement of Cornerstone graft and an anterior cervical plate.

Eatontown, NJ) in all patients except one (Case 5). In this patient, rhBMP-2 was used anteriorly to fill the four cages used in the interspaces between C-3 and C-7.

Follow up ranged from 6 to 9 months, with a mean follow-up duration of 7 months. Fusion was attempted in 18 interspaces, and 17 (94\%) were noted to be fused on the available imaging studies (plain x-ray films and computerized tomography scans) at the most recent follow-up visit. The one patient who did not demonstrate fusion healing (Case 1) continued to show no definitive ossification within the interbody spacer at her 8-month follow-up evaluation (Fig. 3). This patient was subsequently noted to have a good result at her latest clinical follow-up visit.

The sequence of plain x-ray film evidence of fusion maturation consisted of initial blurring irregularity of the absorbable spacer/host bone interface, followed by a distinct column of ossification noted in the midportion of the spacer connecting the contiguous endplates of the fused levels (Figs. 4 and 5). This column of ossification was most pronounced in the patient in Case 5, in whom rhBMP-2 was placed within the interbody fusion cages (Fig. 6). Measurements of soft tissue shadows anterior to the metallic cervical plate showed no significant increase in the patients in whom adequate studies were available. In one patient (Case 8) there was an asymmetrical increased soft tissue shadow anterior to the cervical plate at the level of the C-6 vertebral body, but this had no clinical significance (Fig. 7). In this patient the cephalad soft tis-

TABLE 1

Demographic and clinical data in eight patients who underwent spinal fusion with biosbsorbable implants*

\begin{tabular}{|c|c|c|c|c|c|c|c|c|}
\hline $\begin{array}{l}\text { Case } \\
\text { No. }\end{array}$ & $\begin{array}{c}\text { Age (yrs), } \\
\text { Sex }\end{array}$ & $\begin{array}{l}\text { Date } \\
\text { of Op }\end{array}$ & $\begin{array}{c}\mathrm{FU} \\
(\mathrm{mos})\end{array}$ & Dx & $\begin{array}{c}\text { Levels } \\
\text { Fused }\end{array}$ & $\begin{array}{l}\text { Fusion } \\
\text { Success }\end{array}$ & $\begin{array}{l}\text { Clinical } \\
\text { Outcome }\end{array}$ & Complication \\
\hline 1 & $48, \mathrm{~F}$ & $3 / 12 / 2003$ & 8 & $\mathrm{R}$ & C6-7 & no & good & none \\
\hline 2 & $46, \mathrm{M}$ & $2 / 11 / 2003$ & 8 & M & $\begin{array}{l}\text { C2-3 } \\
\text { C3-4 } \\
\text { C4-5 }\end{array}$ & yes & excellent & none \\
\hline 3 & $64, \mathrm{M}$ & $2 / 14 / 2003$ & 6 & $\mathrm{R} / \mathrm{M}$ & $\begin{array}{l}\text { C5-6 } \\
\text { C6-7 }\end{array}$ & yes & excellent & none \\
\hline 4 & $48, \mathrm{~F}$ & $3 / 25 / 2003$ & 6 & M & C3-4 & yes & good & none \\
\hline 5 & $48, \mathrm{~F}$ & $2 / 18 / 2003$ & 8 & $\mathrm{R} / \mathrm{M}$ & $\begin{array}{l}\mathrm{C} 3-4 \\
\mathrm{C} 4-5 \\
\mathrm{C} 5-6 \\
\mathrm{C} 6-7\end{array}$ & yes & good & none \\
\hline 6 & $46, \mathrm{~F}$ & $2 / 19 / 2003$ & 6 & $\mathrm{R} / \mathrm{M}$ & $\begin{array}{l}\text { C3-4 } \\
\text { C4-5 } \\
\text { C5-6 }\end{array}$ & yes & excellent & none \\
\hline 7 & $60, \mathrm{M}$ & $2 / 26 / 2003$ & 9 & $\mathrm{R} / \mathrm{M}$ & $\begin{array}{l}\text { C5-6 } \\
\text { C6-7 }\end{array}$ & yes & excellent & hematoma \\
\hline 8 & $56, \mathrm{~F}$ & $3 / 03 / 2003$ & 6 & M & $\begin{array}{l}\text { C5-6 } \\
\text { C6-7 }\end{array}$ & yes & excellent & none \\
\hline
\end{tabular}

* Dx = diagnosis; $\mathrm{FU}$ = follow up; $\mathrm{M}$ = myelopathy $\mathrm{R}$ = radiculopathy. 


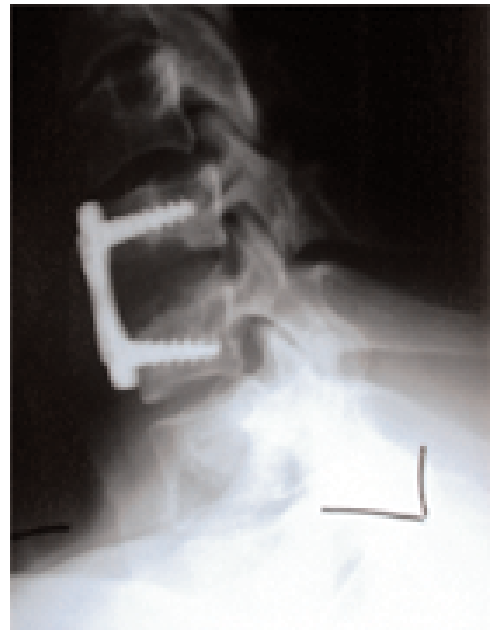

Fig. 3. Lateral plain x-ray film obtained at 8-month follow up demonstrating no obvious bone bridging between the contiguous endplates.

sue measurement at approximately C-5 was $14 \mathrm{~mm}$, at the midplate level (C-6) $20 \mathrm{~mm}$, and at the inferior plate level (C-7) $16 \mathrm{~mm}$. One significant perioperative complication was noted in the patient in Case 7. This patient suffered a symptomatic anterior soft tissue hematoma, which was successfully debrided 1 week after surgical intervention. At their latest follow-up review, three patients were noted to have good clinical outcomes and five had excellent clinical outcomes based on the criteria of Odom, et al. ${ }^{10}$

\section{DISCUSSION}

The evolution of biotechnology has enabled spine surgeons to improve fusion rates by the local administration of rhBMP-2 and to avoid or minimize morbidity related to bone graft harvesting by using synthetic or machined allograft implants. Bioabsorbable implants also

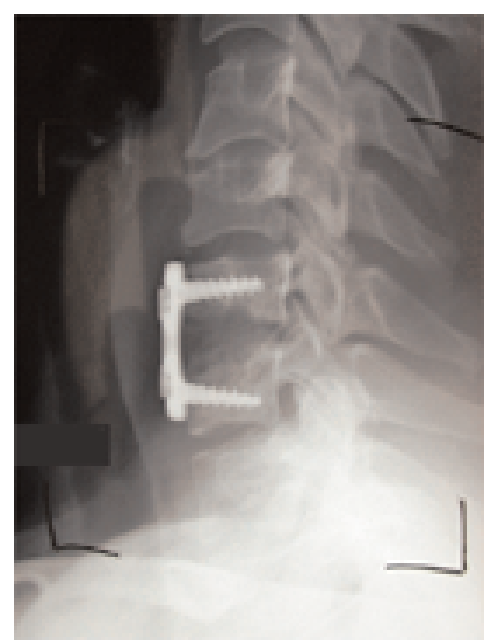

Fig. 4. Lateral plain x-ray film demonstrating early loss of a distinct host bone/interbody spacer interface that was replaced with an irregular blurry interface.

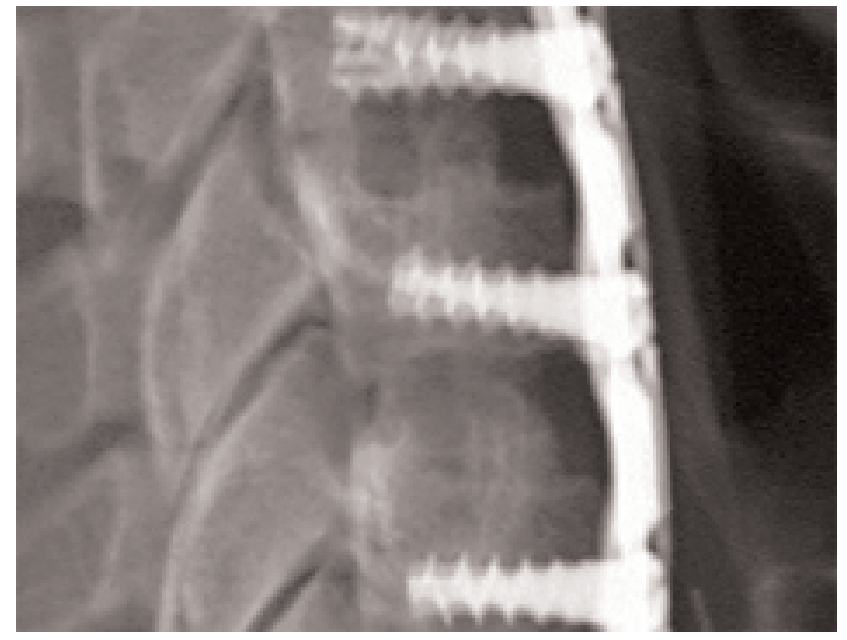

Fig. 5. Lateral plain x-ray film demonstrating a solid anterior fusion with obvious bridging bone (distinct ossified column) between contiguous endplates in a patient in whom a demineralized bone matrix was used as the bone graft replacement in the interbody cages.

provide the potential benefit of improved visualization of the fusion interface. Additionally, through the process of gradual implant resorption, the contained graft substrate can be progressively subjected to loading and can be stimulated to heal.

In this retrospective study we assessed the radiographic and functional outcome in patients who underwent surgical management with an absorbable interbody cage for symptomatic cervical disc degeneration that was causing radiculopathy, myelopathy, or both. In patients with bioabsorbable cages, radiographic and functional outcomes were similar to those reported in a series in which traditional autologous bone grafts were used. ${ }^{2}$ Our study dem-

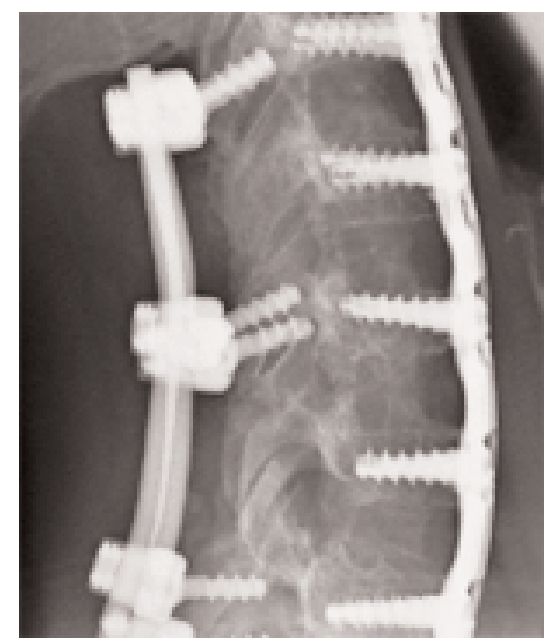

Fig. 6. Lateral plain x-ray film demonstrating a solid anterior fusion with obvious bridging bone (distinct ossified column) between contiguous endplates in a patient in whom rhBMP-2 was added to the interbody cervical spacers. 


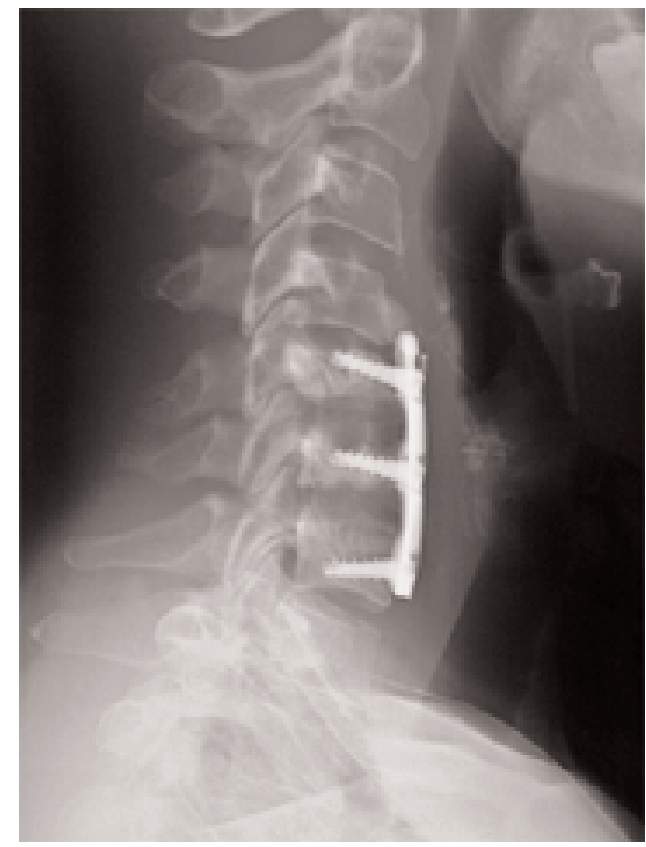

Fig. 7. Lateral plain $x$-ray film demonstrating asymmetrical anterior soft tissue swelling that is most pronounced in the midplate region approximately 6 months postsurgery.

onstrated the ease of assessing the graft/host bone interface in determining fusion status. The byproducts of cage resorption appeared to have no deleterious effect on fusion success: seven (88\%) of eight patients were found to be healed on radiographical studies. There were no cage-related complications, although one patient demonstrated an asymmetrical anterior soft tissue shadow, which appeared to have no clinical sequelae.

In this study we have demonstrated the safety of a bioabsorbable anterior cervical cage. At an early follow up, the implant maintained sagittal alignment and stability while allowing fusion to occur. Inflammatory responses to the components of the bioabsorbable spacers appeared not to be a problem with the current bioabsorbable formulation and application. An interesting observation in this study was the finding of an initial loss and replacement of a distinct host bone/interbody spacer interface with an irregular border, followed later by obvious bridging ossification between the two host endplates at fusion maturation. This process was more pronounced in the one patient in whom rhBMP-2 was added to the four fusion cages. In addition, in our small series of patients, bioabsorbable cages with an anterior metallic cervical plate were able adequately to maintain anterior column height and sagittal alignment.

The clear advantage of a bioabsorbable implant in this application is the lack of an imaging artifact and the implant's progressive resorption, which leaves behind only bone at the fusion site. Theoretically, as the implant degrades over time, a greater amount of stress is transferred to the surrounding bone, which stimulates greater bone remodeling. In addition, the modulus of elasticity of these implants is closer to native bone than the stiffer metallic implants, which lessens the risks of implant migration or subsidence.

\section{CONCLUSIONS}

In this investigation the use of bioabsorbable cages was shown to allow fusion of a decompressed cervical spinal motion segment. There were no appreciable complications specifically related to implant resorption, such as a clinically relevant inflammatory response. This technology will require further prospective study of its use as a carrier for bone graft substitutes to determine whether optimal healing characteristics may eliminate the need for autogenous bone grafting and adjunctive anterior plate fixation.

\section{Disclosure}

Dr.Vaccaro was a consultant for Medtronic Sofamor Danek during the preparation of this manuscript.

The device reported in this series is not approved by the Food and Drug Administration for use in the spine. This device has been cleared by the Food and Drug Administration, however, to maintain the relative position of weak bone tissue in trauma and reconstructive orthopedic procedures when used in conjunction with traditional rigid fixation.

\section{References}

1. Bagby GW: Arthrodesis by the distraction-compression method using a stainless steel implant. Orthopedics 11:931-934, 1988

2. Bohlman HH, Emery SE, Goodfellow DB, et al: Robinson anterior cervical discectomy and arthrodesis for cervical radiculopathy. Long-term follow-up of one hundred and twenty-two patients. J Bone Joint Surg Am 75:1298-1307, 1993

3. Bostman OM: Absorbable implants for the fixation of fractures. J Bone Joint Surg Am 73:148-153, 1991

4. Bucholz RW, Henry S, Henley MB: Fixation with bioabsorbable screws for the treatment of fractures of the ankle. J Bone Joint Surg Am 76:319-324, 1994

5. Christel P, Chabot F, Leray JL, et al: Biodegradable composites for internal fixation, in Winter GD, Gibbons DF, Plenk $\mathrm{H}$ Jr (eds): Biomaterials 1980. New York: Wiley, 1982, pp 271-280

6. Eck KR, Bridwell KH, Ungacta FF, et al: Analysis of titanium mesh cages in adults with minimum two-year follow-up. Spine 25:2407-2415, 2000

7. Eck KR, Bridwell KH, Ungacta FF, et al: Mesh cages for spinal deformity in adults. Clin Orthop 394:92-97, 2002

8. Kandziora F, Schmidmaier G, Schollmeier G, et al: IGF-I and TGF- $\beta 1$ application by poly-(D,L-lactide)-coated cage promotes intervertebral bone matrix formation in the sheep cervical spine. Spine 27:1710-1723, 2002

9. Kuslich SD: Surgical treatment of lumbar degenerative disc disease: axial low back pain, in Vaccaro AR, Betz RR, Zeidman SM (eds): Principles and Practice of Spine Surgery. Philadelphia: Mosby, 2003, pp 365-377

10. Odom GL, Finney W, Woodhall B: Cervical disk lesions. JAMA 166:23-28, 1958

11. Warme WJ, Arciero RA, Savoie FH III, et al: Nonabsorbable versus absorbable suture anchors for open Bankart repair. A prospective, randomized comparison. Am J Sports Med 27: 742-746, 1999

Manuscript received January 15, 2004.

Accepted in final form February 16, 2004.

Address reprint requests to: Alexander R. Vaccaro, M.D., Department of Orthopaedic Surgery, 925 Chestnut Street, 5th Floor, Philadelphia, Pennsylvania 19107; email: Alexvaccaro3@aol.com. 